\title{
The central cluster and X-ray emission from Sgr A*
}

\author{
Robert F. Coker ${ }^{1}$ and Julian M. Pittard ${ }^{2}$ \\ ${ }^{1}$ Los Alamos National Laboratory, Los Alamos, NM 87545, USA \\ ${ }^{2}$ Department of Physics and Astronomy, University of Leeds, Leeds, LS2 9JT, UK \\ email: email: robc@lanl.gov,.jmp@ast.leeds.ac.uk
}

\begin{abstract}
At the centre of the Milky Way is Sgr A*, a putative 3 million solar mass black hole with an observed luminosity that is orders of magnitude smaller than that expected from simple accretion theories. The number density of early-type stars is quite high near Sgr A*, so the ensemble of their stellar winds has a significant impact on the black hole's environment.

We present results of 3D hydrodynamic simulations of the accretion of stellar winds onto Sgr A*. Using the LANL/SAIC code, RAGE, we model the central arc-second of the Galaxy, including the central cluster stars (the S-stars) with orbits and wind parameters that match observations. A significant fraction of the winds from the $\mathrm{S}$ stars becomes gravitationally bound to the black hole and thus could provide enough hot gas to produce the X-ray emission seen by Chandra. We perform radiative transfer calculations on the 3D hydrodynamic data cubes and present the resulting synthetic X-ray spectrum.
\end{abstract}

Keywords. Galaxy: center - stars: winds, outflows - X-rays: ISM

\section{Introduction}

At the dynamical center of the Galaxy lurks Sgr A*, a strong, compact radio source, whose emission is thought to be due to gas accreting onto a $3.6 \times 10^{6} M_{\odot}$ black hole (see Melia \& Falcke 2001 for a review). Chandra X-ray images show a point source, presumably due to thermal bremsstrahlung and up-scattered magnetic bremsstrahlung radio emission, within the $\sim 1$ " astrometric error box of Sgr A*. This point source flares and is surrounded by diffuse $(\sim 0.6$ ") emission in the $2-10 \mathrm{keV}$ Chandra band.

There are dozens of OB stars with $K<17$ mag located within $\sim 1$ " of Sgr A* but only 6 have measured orbital parameters (Eisenhauer et al. 2005). The winds of the stars in this 'Sgr A* IR cluster' contribute to the observed X-ray flux of Sgr A*. So many stars in a small volume results in strong wind collisions and dissipation of kinetic energy. We want to determine how much of the Chandra X-ray flux is from the black hole and how much is directly from colliding stellar winds.

Using the 3D Eulerian code RAGE (Baltrusaitus et al. 1996), we treat the 6 individual OB stars as moving mass $\left(1.0 \times 10^{-6} M_{\odot} \mathrm{yr}^{-1}\right)$ and energy sources $\left(81.4 L_{\odot} \mathrm{yr}^{-1}\right.$; Aerts \& Lamers 2003). Based on the orbits determined from IR observations, we calculate the stars' positions and velocities as a function of time and inject the appropriate mass, momentum, and energy. The volume of solution is taken to be 1.2" (at the GC, 1" $0.04 \mathrm{pc}$ ), the size of the extended Sgr A* emission seen by Baganoff et al. (2003).

We calculate emission and absorption coefficients for each cell and perform a radiative transfer calculation along the line-of-sight to obtain synthetic X-ray images, spectra, and luminosities. We include the gravitational potential from the black hole as well as the underlying stellar cluster. We use boundary conditions such that high velocity gas can escape and external gas can fall in. The calculation discussed here used a resolution 


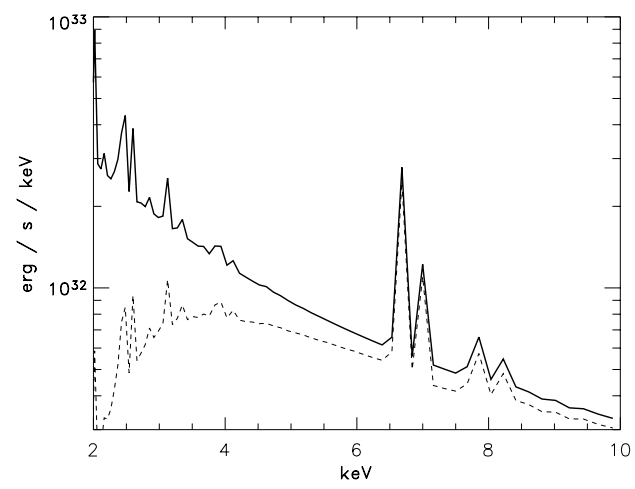

Figure 1. Simulated intrinsic (solid) and absorbed (dashed) $2-10 \mathrm{keV}$ spectrum of the central 1.2" of the Galaxy. The $2-10$ $\mathrm{keV}$ absorbed luminosity, attenuated by a $10^{23} \mathrm{~cm}^{-2}$ column, is $4.7 \times 10^{32} \mathrm{erg} \mathrm{s}^{-1}$. Note the prominent $7 \mathrm{keV}$ Fe line which is also seen in the data.

of $10^{15} \mathrm{~cm}$ with a total of $320^{3}$ cells. The stellar mass, energy, and momentum are injected uniformly in $2.5 \times 10^{15} \mathrm{~cm}$ radius spheres at each star. The ISM is taken to be a $\gamma=5 / 3$ gas with $n=10^{3} \mathrm{~cm}^{-3}$ and $T=10^{4} \mathrm{~K}$ in order to represent the hot, dense GC environment. The central black hole region is 'frozen' (replaced with initial conditions after every time-step) within $\sim 500 R_{g}$ to remove inflowing mass and energy. Optically thin radiative cooling, based on MEKAL cooling rates and assuming solar abundances, is included. We assume an equilibrium temperature of $10^{4} \mathrm{~K}$ so that cold cells slowly heat up due to the hot emission from the young, massive stars in the region.

\section{Results and Discussion}

The colliding wind regions in the simulation reach $\sim 10^{7} \mathrm{~K}$ while the central region attains more than $10^{8} \mathrm{~K}$. The capture radius of these winds is $\sim 0.03 \mathrm{pc}$ so almost all of the gas is bound to the black hole. Wind speeds of $\sim 2000 \mathrm{~km} \mathrm{~s}^{-1}$ (e.g. due to O stars) would be mostly unbound. Since the 6 stars are spectrally identified as B stars, their 'slow' winds should be accumulating in the central 1", as they do in the simulation (although they do reach saturation). With a column density of $\sim 10^{23} \mathrm{~cm}^{-2}$, all of the intrinsic X-ray luminosity of $\operatorname{Sgr} \mathrm{A}^{*}\left(2.4 \times 10^{33} \mathrm{erg} \mathrm{s}^{-1}\right)$ can be produced by just these $6 \mathrm{OB}$ stars; their simulated intrinsic luminosity is $3.3 \times 10^{33} \mathrm{erg} \mathrm{s}^{-1}$. The predicted intrinsic $2-10 \mathrm{keV}$ photon index $(\sim 3.0)$ also matches the observations $(\sim 2.7)$. Although the predicted emission is more compact than observed, including the other two dozen OB stars in the region should extend it (while permitting a lower mass loss rate for the stars). Baganoff et al. (2003) rejected the OB stars as the source of the Sgr A* emission based on Pittard \& Stevens (1997), which required binaries or stellar separations of less than $\sim 10^{14} \mathrm{~cm}$. However, the presence of a potential well enhances the density and temperature such that the $\mathrm{OB}$ stars alone are sufficient to explain the observed quiescent X-ray luminosity of Sgr A*. Thus, accretion models which predict constant significant X-ray emission from within $\sim 500 \mathrm{R}_{g}$ of the black hole Sgr A* may need to be revised.

\section{References}

Aerts, C. \& Lamers, H. J. G. L. M. 2003, A\&A, 403, 625

Baganoff, F. K. et al. 2003, ApJ, 591, 891

Baltrusaitis, R. M. et al. 1996, Physics of Fluids, 8, 2471

Eisenhauer, F. et al. 2005, ApJ, 628, 246

Melia, F. \& Falcke, H. 2001, ARAA, 39, 309

Pittard, J. M. \& Stevens, I. R. 1997, MNRAS, 292, 298 